\title{
2-D Image Diagnostic Technique for Edge Turbulence Using Fast Cameras
}

\author{
N. NISHINO, T. MIZUUCHI ${ }^{1)}$, Z. FENG ${ }^{2)}$, S. KOBAYASHI $^{1)}$, K. NAGASAKI $^{1)}$, H. OKADA $^{1)}$, \\ F. $\mathrm{SANO}^{1)}$, K. KONDO ${ }^{3)}$, Y. NAKASHIMA ${ }^{4)}$, Y. HIGASHIZONO ${ }^{4)}, \mathrm{H}_{\text {. KAWANO }}^{4)}, \mathrm{K}^{4} \mathrm{CHO}^{4)}$, \\ M. SHOJI ${ }^{5)}$, K. SASAKI ${ }^{6)}, \mathrm{K}_{\text {. HANADA }}{ }^{7)}$ and H. ZUSHI ${ }^{7)}$ \\ Graduate school of Engineering, Hiroshima University, Hiroshima 739-8527, Japan \\ ${ }^{1)}$ Institute of Advanced Energy Kyoto University, Kyoto 611-0011, Japan \\ ${ }^{2)}$ Southwestern Institute of Physics, Chengdu, China \\ ${ }^{3)}$ Graduate School of Energy Science, Kyoto University, Kyoto 611-0011, Japan \\ 4) Plasma Research Center, University of Tsukuba, Ibaraki 305-8577, Japan \\ ${ }^{5)}$ National Institute of Fusion Science, Gifu 509-5292, Japan \\ ${ }^{6)}$ Graduate School of Engineering Sciences, Kyushu Univ., Kasuga 816-8580, Japan \\ ${ }^{7)}$ Research Institute for Applied Mechanics, Kyushu Univ., Kasuga 816-8580, Japan
}

(Received 7 December 2006 / Accepted 18 April 2007)

\begin{abstract}
Fast cameras are powerful tool to visualize the edge turbulence in peripheral plasmas. Under Bi-directional collaborations recently in Heliotron $\mathrm{J}$ and GAMMA10 filamentary structures along the magnetic field line were firstly observed with GPI (gas puff imaging) by fast camera. In both machines the filamentary structures had similar stripe pattern in the images and simultaneous measurements of the ion saturation current by electrostatic probes show that the filamentary structures were relatively higher electron density/temperature regions in peripheral plasmas. It is not sufficient to conclude both filamentary structures are the same, however, these phenomena were thought to be related to the energy confinement. Thus the physics mechanism should be solved in the near future.
\end{abstract}

(c) 2007 The Japan Society of Plasma Science and Nuclear Fusion Research

Keywords: 2-d image, fast camera, edge turbulence, Heliotron J, GAMMA10, CPD, ST

DOI: $10.1585 /$ pfr.2.S1055

\section{Introduction}

Recently the progress of the technique of fast cameras is very successfully. In many experimental machines fast cameras are installed to visualize the edge phenomena [1-4]. The edge turbulences in toroidal plasmas were sometimes very similar because many of them were along the magnetic field lines. Not only in toroidal plasmas but also in open field system such as tandem mirror machine GAMMA10 it is very interesting scientific issue to study. Thus, to compare the edge turbulences in various magnetic configurations from the viewpoint of the energy confinement is very important.

Measurements of edge turbulences in helical field and mirror field configurations were performed using fast cameras under several Bi-directional collaborations (between Tsukuba Univ., NIFS, Kyoto Univ., Hiroshima Univ., Kyushu Univ.). The brief summaries of these collaborations are reported in this paper.

\section{Experimental Set Up}

\subsection{Heliotron J}

Heliotron $\mathrm{J}$ is a medium sized Heliotron device, and it has $L=1 / M=4$ helical axis which can form various magnetic configurations [5-7]. The schematic of experimental setup for this experiment is shown in Fig. 1. In the figure, the poloidal cross-section of the standard magnetic configuration of Heliotron $\mathbf{J}[8]$ is also shown. A movable electro-static probe of which head diameter is $22 \mathrm{~mm}$ was installed and it was inserted into the peripheral plasma from a horizontal port to localize the observation area for a fast camera. Movable retractable Langmuir probes $\left(1.5^{\phi} \times 3 \mathrm{~mm}^{L}\right.$ molybdenum probe tips $)$ are embedded in this head. The fast camera FX-K4 (NAC image technology, http://www.nacinc.com/) views this probe from the upper horizontal port at the same toroidal section.

\subsection{GAMMA10}

GAMMA10 is a (an effectively axisymmetrized minimum-B anchored) tandem mirror with thermal barrier at both end-mirrors $[9,10]$. The device consists of a centralmirror region, anchor-regions with minimum-B configuration (using baseball coils), and plug/barrier regions with 


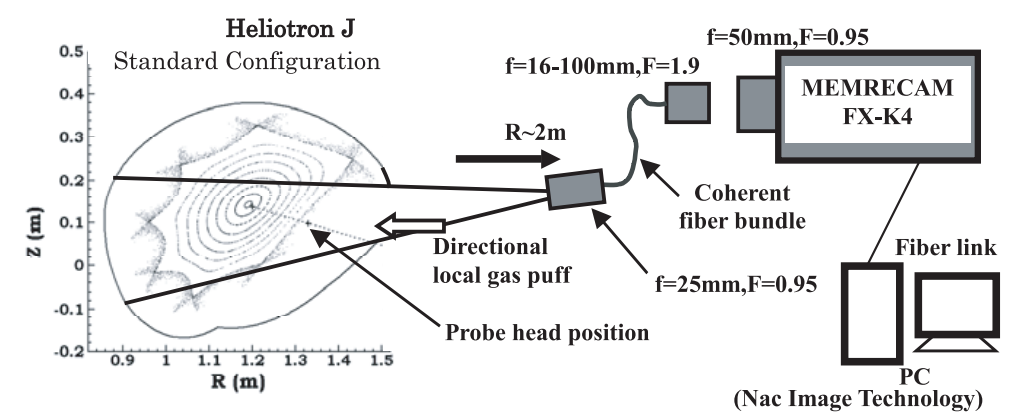

Fig. 1 Schematic view of the poloidal cross-section of Heliotron J plasma and camera system Gas puff, movable probe and object lens are installed at the same toroidal section.

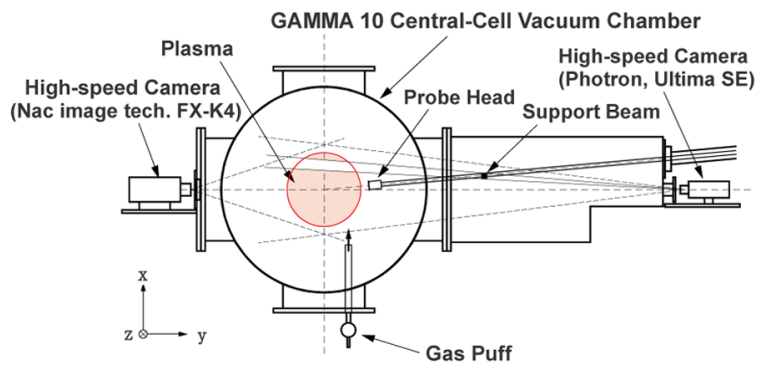

Fig. 2 Schematic view of the cross-section of the central-cell GAMMA 10 plasma Gas puff for imaging technique was installed at the bottom left.

axisymmetric mirrors. In this experiment the gas puff in the central-cell (the center of the central-mirror region) was used to visualize plasma periphery. Figure 2 shows the schematic of the GAMMA 10 central-cell plasma and the location of the fast camera (left side). The gas puff was located at the bottom port near the central-cell mid plane.

\section{Results and Discussion}

For the edge turbulence measurement there are three Bi-directional collaborations. However, the GPI experiment using the fast camera in CPD (ST in Kyushu Univ.) is not in time. Therefore, in this section the results of GPI experiments in Heliotron J and GAMMA 10 are described in turn briefly.

\subsection{Heliotron J}

Figure 3 shows the ion saturation current during ECH discharge with GPI in Heliotron J standard configuration. Gas puff was injected after H-mode. Figure 4 shows the camera image during GPI with the speed of 80,000 frames per second (FPS). Turbulence of the filamentary structure along the magnetic field line was shown clearly.

Figure 5 shows two-dimensional phase image of which frequency is $7.5 \mathrm{kHz}$ by time-dependent FFT analysis of each pixel data from camera image. (The original figures use color, but the print is $\mathrm{B} / \mathrm{W}$. Therefore, the reader should be careful to see these images.) Filamentary struc-

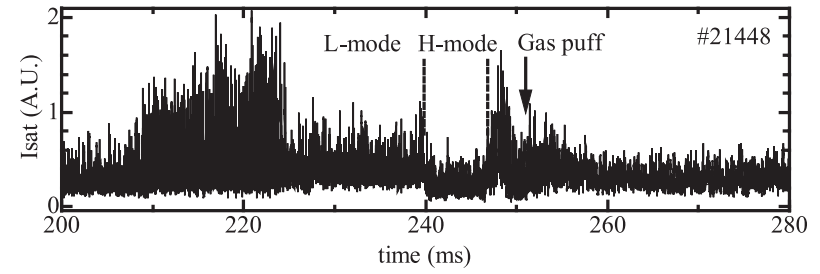

Fig. 3 Time evolution of the ion saturation current for ECH discharge in the standard configuration.

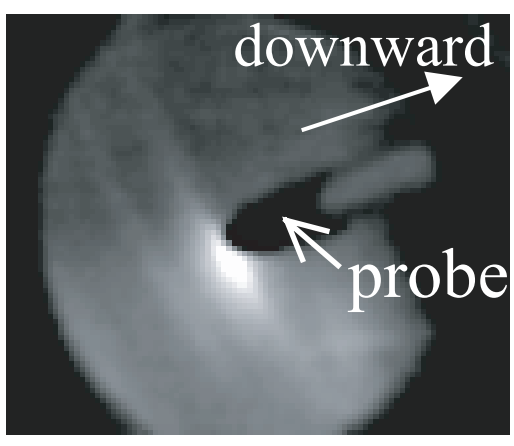

Fig. 4 Typical image during GPI (\#21448).

ture was shown in L-mode in Fig. 5 (a), but a little structure was recognized in H-mode in Fig. 5 (d), even though the light intensity was not sufficient. During L-H transition and $\mathrm{H}-\mathrm{L}$ transition the light intensity increased within short period ( $<1 \mathrm{~ms})$ and every points had almost the same phase such as Fig. 5 (b). Just after L-H transition and just before $\mathrm{H}-\mathrm{L}$ transition relatively wide structures were recognized in Fig. 5 (c) and (e). Both wide structures rotated from upward to downward in the camera images. Similar rotation just after H-mode was already observed in our previous work [11], and the rotation speed was $5-6 \mathrm{kHz}$. This time rotation speed is around $8 \mathrm{kHz}$ from phase images. After $\mathrm{H}$-mode during GPI relatively narrow structure was shown in the phase image in Fig. 5 (f), which was already shown in real image of Fig. 4. Each filamentary structure moved 


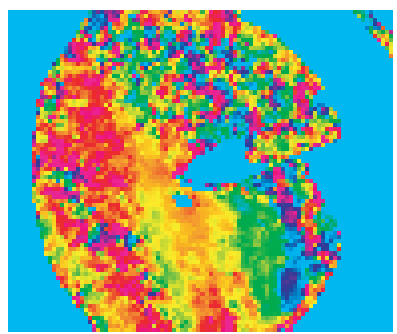

(a) L-mode

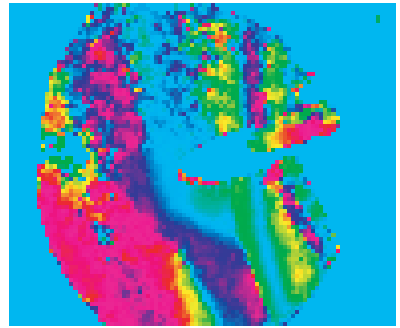

(c) Just after L-H transition

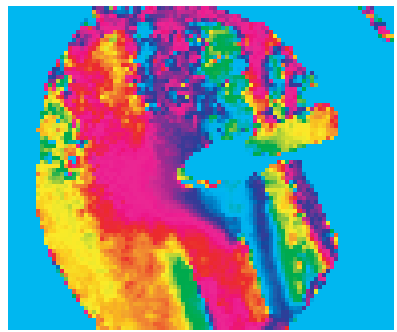

(e) Just before $\mathrm{H}-\mathrm{L}$ transition

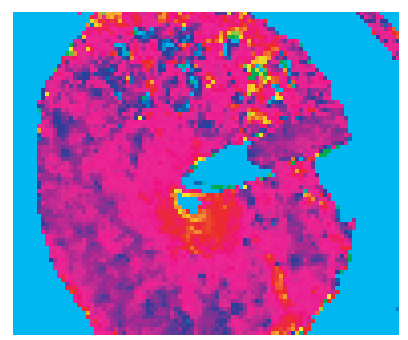

(b) L-H transition

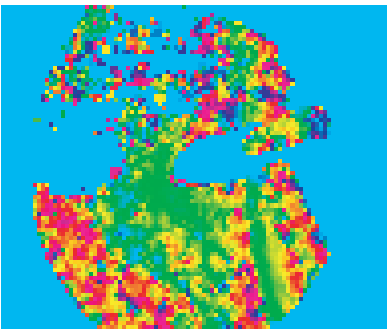

(d) H-mode

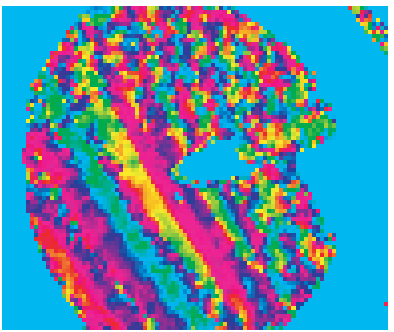

(f) GPI
Fig. 5 Phase images during ECH discharge of the same shot in Fig. 3 and 4 (\#21488).

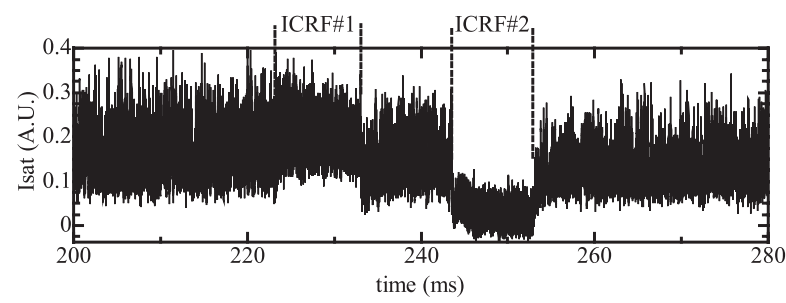

Fig. 6 Time evolution of the ion saturation current for ICRF heating discharge in the standard configuration.

across the magnetic field lines during GPI. The phase images with the frequency range of $5 \mathrm{kHz}$ to $20 \mathrm{kHz}$ were similar in this shot.

In high electron density regime of $>2.6 \times 10^{19} \mathrm{~m}^{-3}$ typical lifetime of each filament in the camera images was $600 \mu \mathrm{s}$ [12]. However, in low density plasmas each filament moved quickly and it was impossible to find the birth and disappearance position of filamentary structure. With comparison of the ion saturation current and camera data it is inferred that the filamentary structure is relatively high electron density and/or temperature region in peripheral plasma.

Figure 6 shows the ion saturation current during typ-

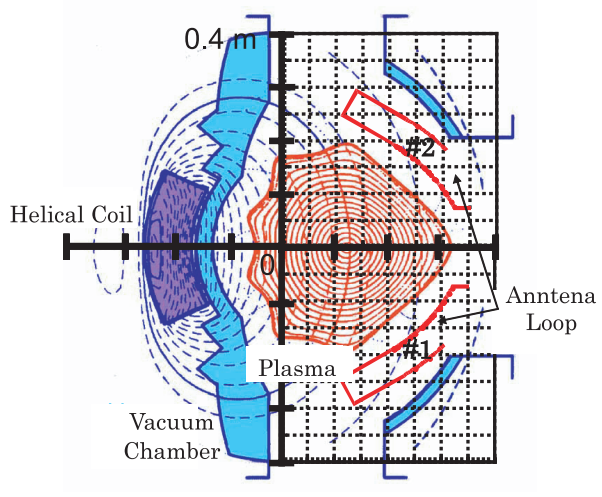

Fig. 7 Schematic view of ICRF antenna and the magnetic surface of the standard configuration in Heliotron J.

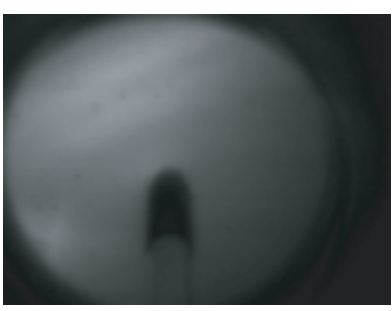

(a) Just before ICRF\#2

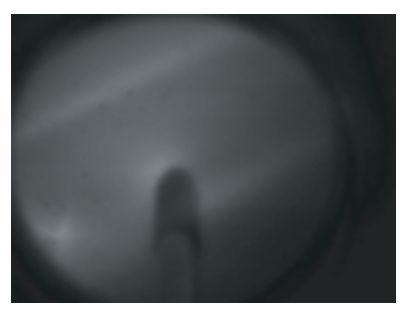

(b) During ICRF\#2
Fig. 8 Images before and during ICRF \#2.

ical ICRF discharge in Heliotron J standard configuration. During ICRF the increase and/or decrease of the ion saturation current were often observed. Figure 7 shows the poloidal cross-section of the ICRF antenna and LCFS of the standard configuration. At the same time the suppression of the filamentary structure was observed in the camera image only during ICRF\#2. Figure 8 shows typical camera images of ICRF discharge. Before ICRF\#2 the filamentary structure was seen, and it moved downward direction. During ICRF\#2 the filamentary structure was not seen, instead of it two feet lines of the edge magnetic field were seen clearly. These lines are consistent with threedimensional view of the magnetic field line of Heliotron $\mathrm{J}$, and it did not moved during ICRF\#2. It was unclear that the relationship between the suppression of the filamentary structure and the decrease of the ion saturation current. The ratio of local $\mathrm{H} \alpha$ intensity near the probe and the ion saturation current is almost proportional to the local neutral density. This ratio during ICRF\#2 increased in comparison with before and after ICRF\#2. This means the local neutral density near the probe tip increased during ICRF\#2. ICRF\#2 used upper antenna in Fig. 7, and the whisker field lines near the antenna come to be that in front of the camera image. Therefore, it might be considerable that the antenna interact peripheral plasma stimulated by ICRF. The reason of the increase and decrease of the ion saturation current was not fully understood, however, the particle confinement may be worse or better in the peripheral plasma re- 
gion of these period, respectively. Even now any evidence on good/bad energy confinement during ICRF discharges, there might be the possibility of the change of the RF accessibility due to the non-linear heating effect by ICRF. This issue should be treated carefully due to the effect of the uncontrollable recycling. The increment and decrement of the ion saturation current were apparently proportional to the net ICRF power. By FFT analysis the frequency peak of $90-100 \mathrm{kHz}$ was found in the ion saturation current during ICRF\#2. This peak was recognized clearly with the decrease of the ion saturation current. These phenomena should be solved in the near future.

\subsection{GAMMA 10}

In GAMMA 10 plasma is initiated by two plasma guns from both ends and then the main plasma is started up with two ICRF waves (excited in the central region and anchor region, respectively). End-loss particles along the magnetic field lines are confined by the plasma confining potentials produced with ECH in both plug/barrier regions. Usually the ion temperature is rather higher than the electron temperature and it is necessary to reduce the energy loss due to ion-electron heat conduction. Recently ECH in the central region $(\mathrm{C}-\mathrm{ECH})$ was applied to achieve high electron temperature.

GPI experiment with C-ECH and without C-ECH was performed and it was successful to take the images of the edge turbulence. Figure 9 shows typical waveform of GAMMA 10 plasma of GPI experiment. Figure 10 shows typical GPI image with $\mathrm{C}-\mathrm{ECH}$, and Fig. 11 shows typical GPI image without C-ECH. In both figures the bright emission regions were seen near the gas puff location at the bottom, and there were filamentary structures along the magnetic field lines in Fig. 10. The structure of edge plasma was unclear in Fig. 11. The difference condition between both shots was only C-ECH. According to our model of filament [13] it is due to C-ECH un-uniform heating along the magnetic field line. However, the experimental evidence was not sufficient to claim the validity of our model up to now. This will be next our work in the short term.

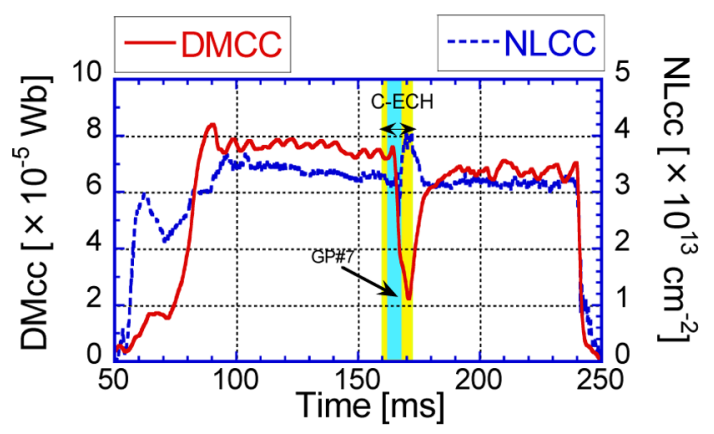

Fig. 9 Typical discharge waveform of GPI experiment during ECH DMcc is the diamagnetic signal and NLcc is the line integral of the electron density.
Using FFT analysis of the pixel point data of which emission is very bright during GPI, the broad peak frequency of $6.5 \mathrm{kHz}$ was found. Figure 12 shows two-dimensional phase image of $6.25 \mathrm{kHz}$ frequency component by

time-dependent FFT analysis of each pixel data using 32 frames. This shot was with $\mathrm{C}-\mathrm{ECH}$, and the structure along the magnetic field lines were seen much clearly. These stripe patterns rotated from upward to downward first. About $165 \mathrm{~ms}$ the rotation stopped, then the plasma rotated reverse direction. This interesting behavior was believed to relate the diamagnetic signal. During GPI the electron density also increased but the diamagnetic signal decreased. This shows the temperature decreased during GPI. In GAMMA 10 plasma the ion temperature is 5$10 \mathrm{keV}$ and the electron temperature is several hundred $\mathrm{eV}$. Therefore, it is natural thought that the diamagnetic sig-

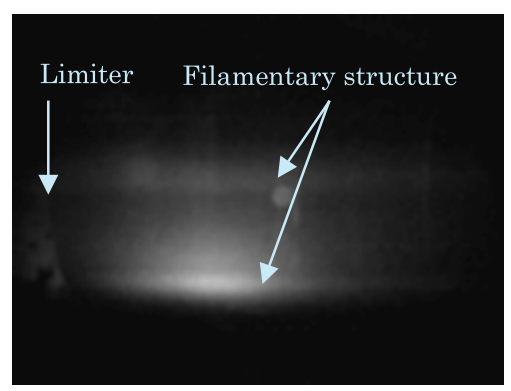

Fig. 10 Image of GAMMA 10 plasma during GPI with C-ECH.

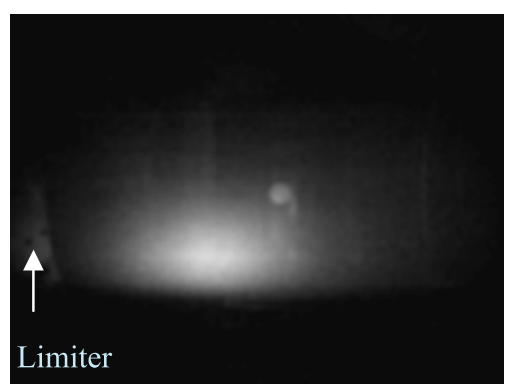

Fig. 11 Image of GAMMA 10 plasma during GPI without C$\mathrm{ECH}$.

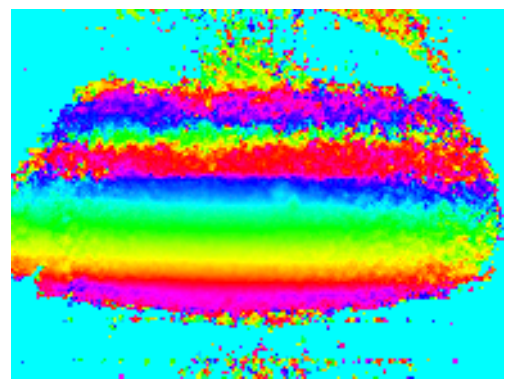

Fig. 12 Two-dimensional phase image of $6.5 \mathrm{kHz}$ component by time-depend FFT during GPI in GAMMA 10 plasma. 
nal shows almost the ion diamagnetic signal. It is possible to say that much gas by GPI increase charge-exchange loss and the ion temperature decreased, however, this decrement of diamagnetic signal is not fully understood. The low ion temperature plasma in GAMMA 10 usually has positive $E r$ and plasma rotate $E \times B$ drift direction. Therefore, the rotation reverse means that low temperature plasma is produced by GPI. Since the electron density is below $1 \times 10^{19} \mathrm{~m}^{-3}$, the light emission in GAMMA 10 plasma is smaller than that of the other toroidal plasma. Therefore, it is necessary to provide much gas puff quantity to plasma column to get the images of the light emission by our fast camera. From this viewpoint this time GPI is not good condition in GAMMA 10 plasma. However, it was the first time to attempt to visualize the edge turbulence.

Under Bi-directional collaborations similar measurements will be performed in CPD (ST machine in Kyushu University) soon. Therefore, in the near future it is possible to compare the edge turbulences in various magnetic configurations. Information on the edge turbulences should have shed much new light on the complex nature of good confinement mode, and it is ultimate aim of this study.

\section{Conclusion/Summary}

Fast camera measurements were performed in $\mathrm{He}$ liotron $\mathrm{J}$ and GAMMA10 to visualize the plasma edge turbulence. In both machine filamentary structures along the magnetic field line were observed. In phenomenology the characteristics of these phenomena are similar by the cam- era images, but it is further question that physics mechanism of both phenomena are the same. Similar measurement will be performed very soon in CPD (ST). The comparison of those data should be quite interesting.

\section{Acknowledgments}

The authors acknowledge NIFS for helping Bidirectional collaboration. N.N thanks to S.Nishino for continues encouragement.

[1] S.J. Zweben, S.S. Medley, J. Nucl. Mater. 145-147, 250 (1987).

[2] S.J. Zweben, R.J. Maqueda, D.P. Stotler et al., Nucl. Fusion 44, 134 (2004).

[3] S.J. Zweben, D.P. Stotler, J.L. Terry et al., Phys. Plasma 9, 1981 (2002).

[4] N. Nishino, K. Takahashi, H. Kawazome et al., J. Plasma Fusion Res. 80, 179 (2004).

[5] T. Obiki, T. Mizuuchi, K. Nagasaki et al., Nucl. Fusion 41, 883 (2001).

[6] T. Obiki, T. Mizuuchi, H. Okada et al., Nucl. Fusion 44, 47 (2004).

[7] T. Mizuuchi, W.L. Ang, Y. Nishioka et al., J. Nucl. Mater. 313-316, 947 (2003).

[8] T. Obiki et al., Plasma Phys. Control. Fusion 42, 1151 (2000).

[9] M. Inutake et al., Phys. Rev. Lett. 55, 939 (1985).

[10] T. Cho et al., Trans. Fusion Technol. 47, No.1T 9-16 (2005).

[11] N. Nishino et al., J. Nucl. Mater. 337-339, 332 (2005).

[12] N. Nishino et al., to be published in J. Nucl. Matter.

[13] N. Nishino, Nucl. Fusion 46, S568 (2006). 